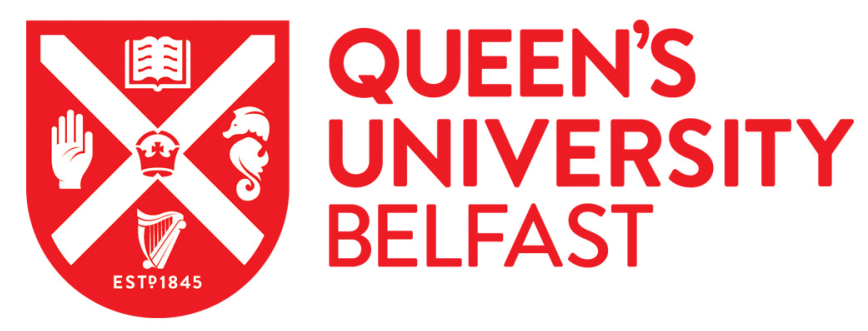

\title{
Deterministic Switching in Bismuth Ferrite Nanoislands
}

Morelli, A., Johann, F., Burns, S. R., Douglas, A., \& Gregg, J. M. (2016). Deterministic Switching in Bismuth Ferrite Nanoislands. Nano Letters, 16(8), 5528-5234. https://doi.org/10.1021/acs.nanolett.6b02311

\section{Published in: \\ Nano Letters}

\section{Document Version:}

Peer reviewed version

Queen's University Belfast - Research Portal:

Link to publication record in Queen's University Belfast Research Portal

\section{Publisher rights}

This document is the Accepted Manuscript version of a Published Work that appeared in final form in Nano Letters, copyright 2016 ( American Chemical Society after peer review and technical editing by the publisher. To access the final edited and published work see http://pubs.acs.org/doi/abs/10.1021/acs.nanolett.6b02311

\section{General rights}

Copyright for the publications made accessible via the Queen's University Belfast Research Portal is retained by the author(s) and / or other copyright owners and it is a condition of accessing these publications that users recognise and abide by the legal requirements associated with these rights.

Take down policy

The Research Portal is Queen's institutional repository that provides access to Queen's research output. Every effort has been made to ensure that content in the Research Portal does not infringe any person's rights, or applicable UK laws. If you discover content in the Research Portal that you believe breaches copyright or violates any law, please contact openaccess@qub.ac.uk. 


\section{Deterministic switching in bismuth ferrite}

\section{nanoislands}

Alessio Morelli ${ }^{\dagger, *}$, Florian Johann ${ }^{\ddagger, s}$, Stuart R. Burns ${ }^{\dagger}, \|$, Alan Douglas ${ }^{\dagger}, \mathrm{J}$. Marty Gregg ${ }^{\dagger}$

${ }^{\dagger}$ Centre for Nanostructured Media, School of Mathematics and Physics, Queen's University Belfast, University Road, Belfast BT7 1NN, United Kingdom

${ }^{\ddagger}$ Max Planck Institute of Microstructure Physics, D-06120 Halle, Germany

KEYWORDS

Bismuth ferrite, multiferroics, nanoislands, polarization switching, scanning probe microscopy 


\section{ABSTRACT}

We report deterministic selection of polarization variant in bismuth $\mathrm{BiFeO}_{3}$ nanoislands via a two-step scanning probe microscopy procedure. The polarization orientation in a nanoisland is toggled to the desired variant, after a reset operation, by scanning a conductive atomic force probe in contact over the surface while a bias is applied. The final polarization variant is determined by the direction of the inhomogeneous in-plane trailing field associated with the moving probe tip. This work provides the framework for better control of switching in rhombohedral ferroelectrics and for a deeper understanding of exchange coupling in multiferroic nanoscale heterostructures, towards the realization of magnetoelectric devices.

\section{MAIN TEXT}

Issues associated with heat management and power consumption have ubiquitous significance in the development of microelectronic devices ${ }^{1}$. The use of electric fields alone, rather than currents or magnetic fields, to control device operation is attractive in this context ${ }^{2}$, as dramatic reductions in heat generation and power consumption can, in principle, be achieved. Such considerations have revived interest in multiferroic magnetoelectrics, in which magnetism can be controlled via electric field $\mathrm{d}^{3,4}$. However, the scarcity of room temperature single phase multiferroics with high magnetoelectric coefficients and large electric and magnetic dipolar moments has pushed research towards an investigation of composite materials, in which the order parameters are coupled via proximity effects occurring at the interface ${ }^{5-7}$.

The single phase room temperature ferroelectric antiferromagnetic oxide, bismuth ferrite (BFO), has been widely studied in recent years ${ }^{8}$. In conjunction with $\mathrm{Co}_{0.90} \mathrm{Fe}_{0.10}$ (CoFe) in multiferroic composite heterostructures, it has been used to demonstrate electric-field driven 
magnetization rotation ${ }^{9-12}$. This is the result of a double magnetoelectric coupling mechanism involving: firstly, BFO internal coupling, in which anti-ferromagnetic planes rotate following electric-field induced polarization switching ${ }^{13}$; secondly, interfacial exchange coupling by which in-plane magnetization in CoFe is linked to the projection of the antiferromagnetic planes on the (001) interface ${ }^{11,14}$. Due to the rhombohedral structure of BFO, there are eight possible equivalent pseudocubic $<111>_{\text {pseudocubic }}$ polar directions, with three possible switching phenomena classified by the angle between initial and final polarization variant $\left(71^{\circ}, 109^{\circ}\right.$ and $180^{\circ}$ ). By geometrical considerations, rotation of the projection of the antiferromagnetic ordering on the (001) surface occurs only for in-plane $71^{\circ}$ and out-of-plane $109^{\circ}$ polarization switching ${ }^{13}$. The former has actually been verified to induce magnetization rotation ${ }^{9}$ in a mesoscopic heterostructure with planar electrodes. Unexpectedly, even $180^{\circ}$ switching has resulted in magnetization rotation (actually inversion) in a mesostructure with a perpendicular electrode configuration, explained as being a two-step switching sequence composed of in-plane $71^{\circ}$ followed by out-of-plane $109^{\circ}$ switching ${ }^{15}$.

Given that only determinate switching procedures in BFO may lead to magnetization rotation, there is a need to gain a deeper understanding over polarization switching in rhombohedral BFO, with the aim of achieving full deterministic control. Polarization switching in rhombohedral ferroelectrics can often be complicated because of the relatively large number of energetically equivalent polar variants available ${ }^{16}$ : switching using electric fields along pseudocubic $<100>$ directions, for example, results in four energetically equivalent switched states.

Deterministic selection has been achieved by use of specific electrode design, capitalizing on the characteristics of the specific material system: rotation of polarization variant between two possible states in mesostructured single-variant $\mathrm{BFO}^{17}$, and stripe domain configuration $180^{\circ}$ 
reversal in two-variant $\mathrm{BFO}^{12}$. To gain a better understanding of the process at the nanoscale, switching investigations have been performed by Piezoresponse Force Microscopy (PFM). The movement of a biased probe tip over the sample surface has been used to break the symmetry of the field experienced by the material, so that the final state adopted is strongly influenced by the direction of the in-plane component of the trailing field. Trailing fields have been employed to control domain writing with a certain degree of success for a range of materials ${ }^{18-20}$, including $\mathrm{BFO}^{21-23}$. Control of predefined stripe domain patterns ${ }^{21}$ and of ferroelectric polarization ${ }^{22}$ has been achieved; however the influence of the surrounding matrix in continuous ferroelectric films has led to problems in retention at the nanoscale level. A higher degree of control has been obtained in two-variant films with polarization selection in sub-micron areas remaining stable for the duration of the experiment ${ }^{23}$.

The investigation of deterministic polarization switching in BFO has not been previously performed on free-standing nanoislands, despite the fact that device miniaturization would require an understanding and verification of the phenomenon in this kind of geometry. There could even be advantages to switching in patterned media, as reduced lateral dimensions have been demonstrated to improve retention ${ }^{17}$.

In this letter we present PFM investigations concerning polarization switching in BFO nanoislands by predominantly [001] oriented electric fields. We demonstrate that sub-micron lateral size and limited constraint from surrounding material can allow polarization to be effectively toggled among any of the four variants pointing towards the bottom electrode, a feat not achieved in previous research. Each polar orientation is selected, after a 'reset' operation, via the raster scanning direction of the Scanning Probe Microscopy (SPM) probe. With the aid of electric field simulations, we demonstrate that the operation is made possible by the 
inhomogeneous electric fields generated at the PFM probe-tip: a transverse field is confined to a small volume at the edges of the tip-sample contact area which allows the in-plane component of the polarization to be controlled during the out-of-plane switching procedure.

Moreover, we report that a quadrupole configuration is stabilized by switching the polarization towards the top surface of the BFO nanoislands; in previous studies on continuous films, this dipole pattern had been highly unstable.

Experiments were performed on BFO islands fabricated from a parent (001) BFO thin film (30nm) grown by Pulsed Laser Deposition on $\mathrm{SrTiO}_{3}$ with a $30 \mathrm{~nm} \mathrm{SrRuO}_{3}$ buffer layer as a bottom electrode. Details on film growth are described elsewhere ${ }^{24}$. Fabrication of the islands from the BFO parent film was performed by a mask-assisted Focused Ion Beam (FIB) milling procedure $^{25}$, which has been demonstrated to produce islands which retain their ferroelectric properties with minimal fabrication-induced damage ${ }^{26}$. The milling procedure yielded a milled square area $\left(5 \times 5 u^{2}\right)$ containing a number of BFO islands about $400 \mathrm{~nm}$ in diameter and $25 \mathrm{~nm}$ in height (Figure 1).
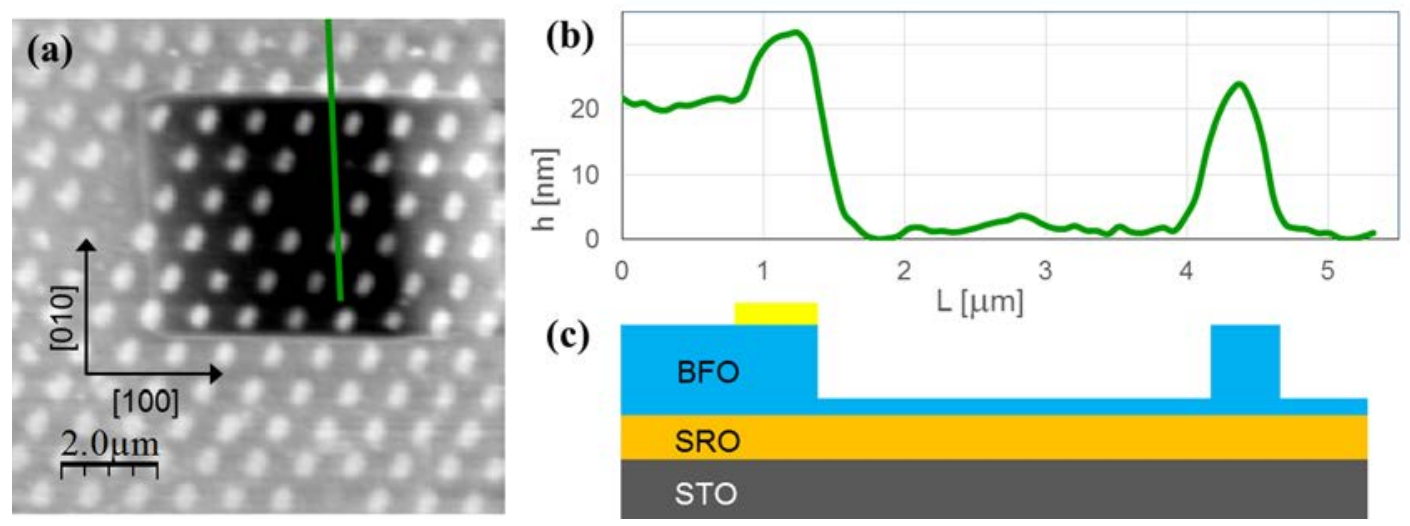

Figure 1. Topography (a), line profile (b), and schematic diagram (c) of the patterned portion of the BFO film in which nanoislands of BFO have been produced. 
Investigations to reveal domain configurations were performed by vector PFM, which allows identification of polarization variants by consecutive scans of the same area with the probe cantilever oriented along two orthogonal $\operatorname{axes}^{27}$. The parent film has a stripe-like ferroelectric domain configuration (top areas in Figure 2a-e), with a predominance of variants with downward out-of-plane components (i.e. polarization vectors pointing towards the bottom electrode). The initially observed domain configurations in the nanoislands are clearly inherited from the parent film, since the BFO ferroelectric Curie temperature $\left(\mathrm{T}_{\mathrm{c}} \sim 1100 \mathrm{~K}\right)$ is not exceeded during any step of the fabrication procedure (Figure 2e). Piezo-hysteresis loops ${ }^{28}$ measured over a virgin area of parent film and the middle of an island (Figure S1) confirm preservation of ferroelectricity with minimal change in imprint resulting from the island patterning process.

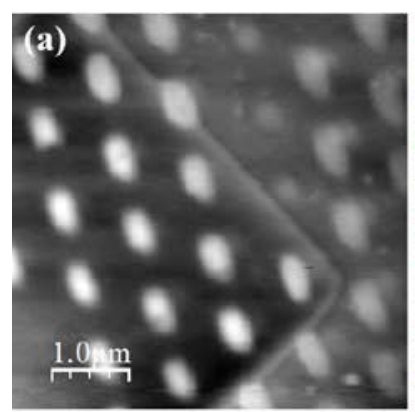

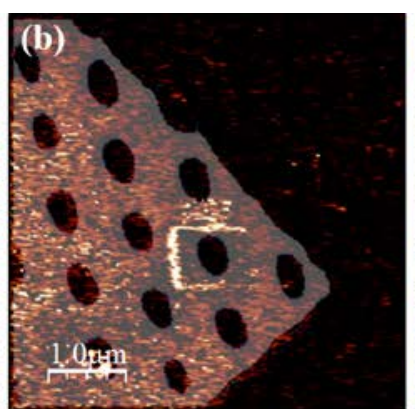

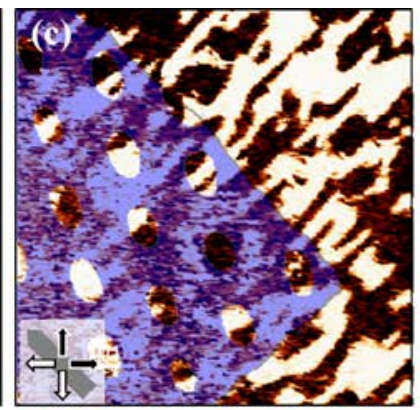

(e)
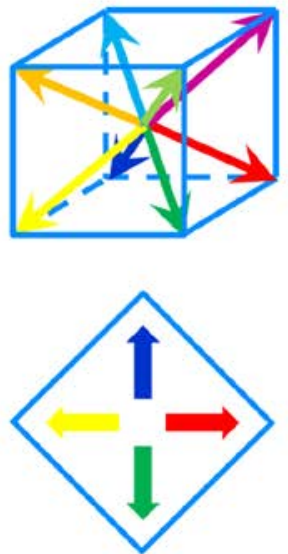

(f)
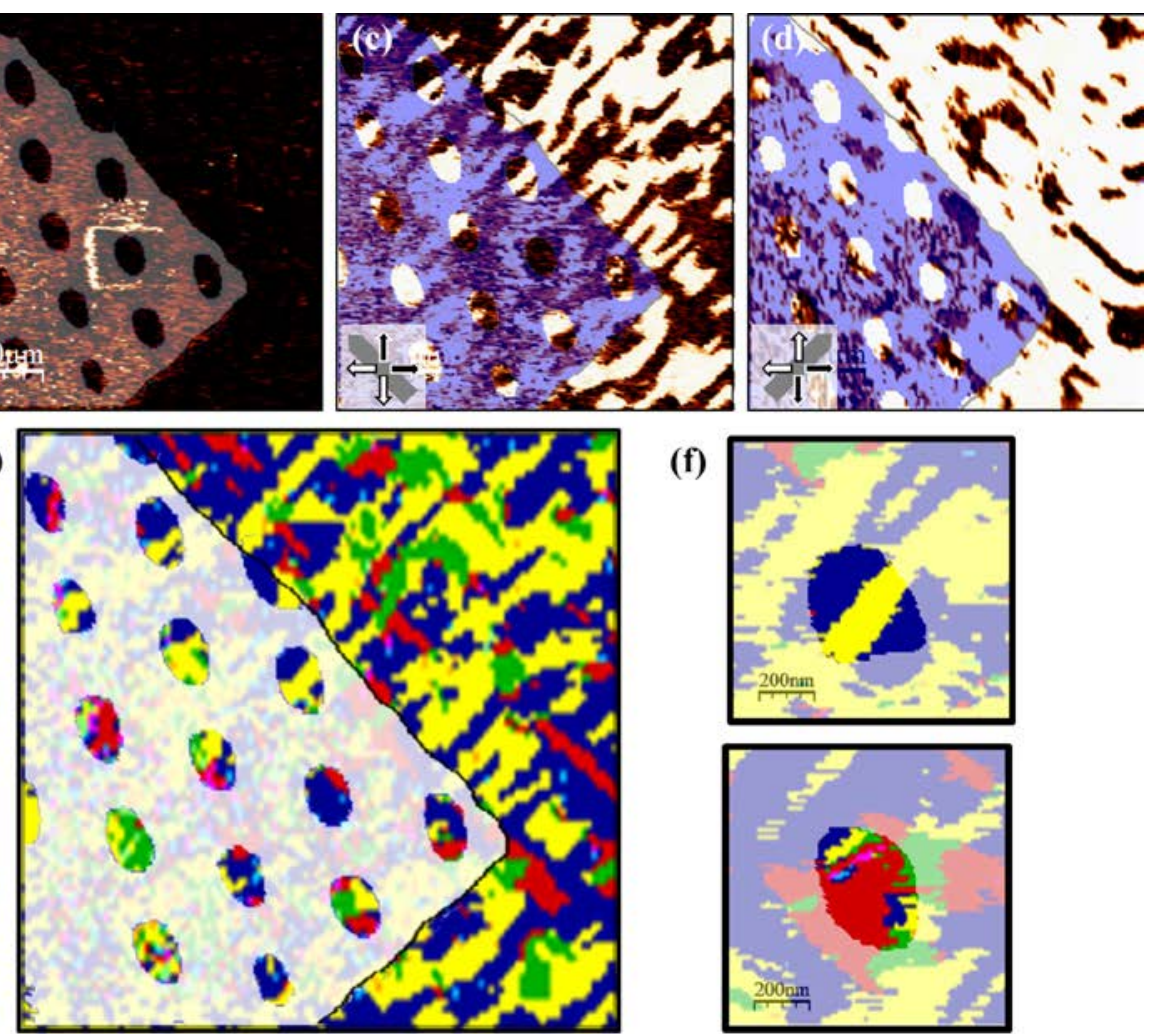

Figure 2. Topography (a); vertical PFM phase (b); lateral PFM phase with cantilever axis along [0-10] (c) and [-100] (d) of a portion of milled area. Domain color map with different 
polarization orientations inferred from vector PFM (e), with 3 dimensional and top-view planar color coding (left), and a magnified image of the domain configurations in two islands from a different region of the machined area (f). A topographic mask has been superimposed onto the PFM images and Vector PFM maps in order to identify the island positions. Inset diagrams in (c) and (d) represent cantilever orientation with colour coding for Lateral PFM image interpretation.

Switching experiments were performed using PFM, scanning the grounded probe over the surface of the island under investigation while the bottom electrode was biased (Figure 3a,b). The expected control over the switched polar orientation is understood by considering the inhomogeneous nature of electric field in the proximity of the PFM probe, combined with its movement. Application of an out-of-plane electric field across rhombohedral (001) BFO leads to reversal of the out-of-plane component of polarization, with four available variants nearly energetically equivalent ${ }^{16}$. The field generated between the PFM probe and the bottom electrode has an inhomogeneous normal component in the volume underneath the contact area, and a radial transverse component around it. When the probe is not moving, the normal field induces out-of-plane polarization reversal, and in-plane polarization reorientation with opposite senses either side of the contact area. Removal of the field leads to an energetically unfavorable charged domain wall, which encourages relaxation of domains into a different configuration, determined by mechanical and electrostatic boundary conditions ${ }^{22}$. Selection between the available variants can be achieved by moving the probe over the surface, hence breaking the symmetry of the transverse field. The moving probe creates a virtual 'trailing field', the direction of which is opposite to its movement (Figure 3). Slow raster axis scanning along [100] or [010] leads to a multidomain configuration, as two out of the original four $<111>$ polar variants are still 
energetically equivalent under the influence of the trailing field. On the other hand, slow raster axis scanning along [110] or [1-10] breaks the symmetry in favour of only one of the available four variants and hence selects it.

(a)

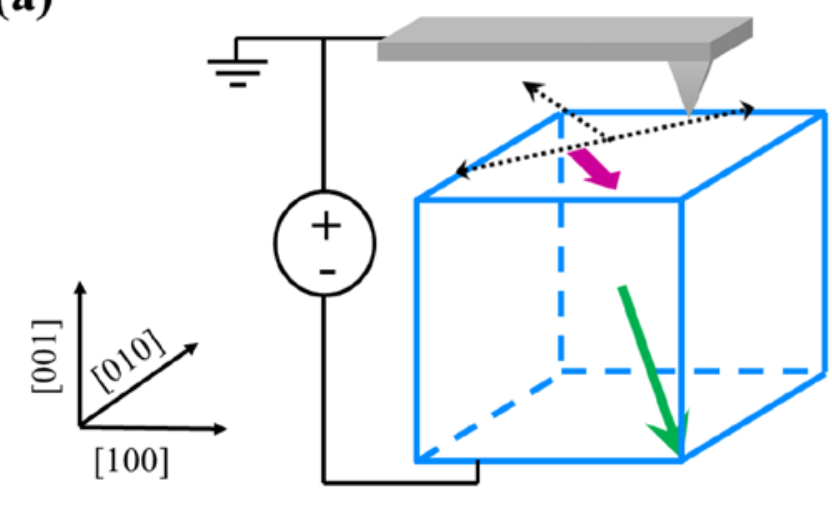

(b)

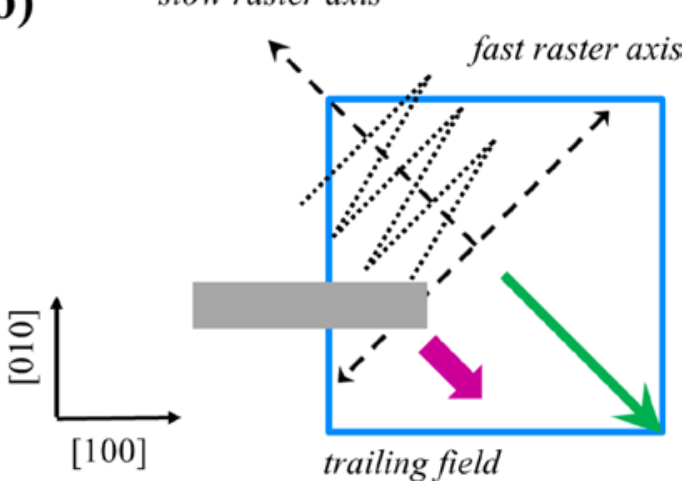

Figure 3. Schematic of the switching experiment with probe raster scanning over a unit cell, with trailing field (fat arrow) and polarization variant selected by it (a), and top view (b) of the same.

Reversal of as grown island out-of-plane polarization from downwards to upwards requires a positive DC bias (typically $+6 \mathrm{~V}$ ). Irrespective of the slow raster axis direction used during poling, the resulting island domain configuration is always multidomain (Figure 4b,c,d) with outof-plane components upwards. Three-dimensional domain mapping reveals a quadrupole or antivortex pattern (Figure 4e,f) often results. This may be of interest for the investigation of topological patterns ${ }^{29-32}$.

The virgin domain configuration displaying downwards polarization (Figure 2b) and the measured imprint both in films and islands (Figure S1) indicate efficient polarization screening at the bottom interface, rendered possible by free carriers in the electrode ${ }^{33,34}$. On the other hand, at the top surface and sidewalls of the island screening is not as efficient, and depolarizing fields 
are inevitable, which contribute to the multidomain state found when polarization points upwards $^{31}$.
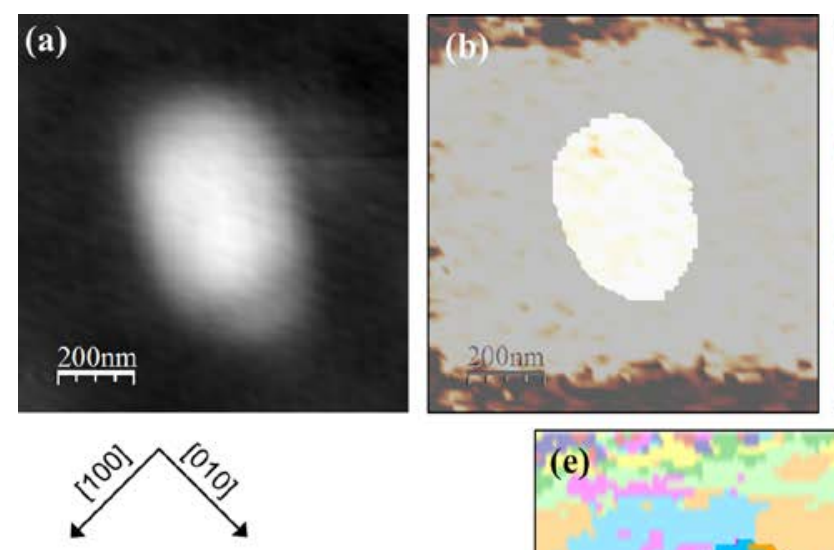

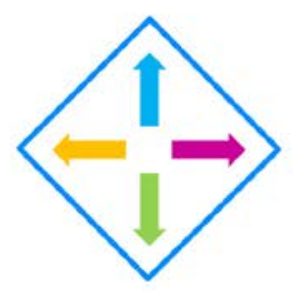

(e)

$\frac{200 \mathrm{~nm}}{11 \mathrm{~T}}$
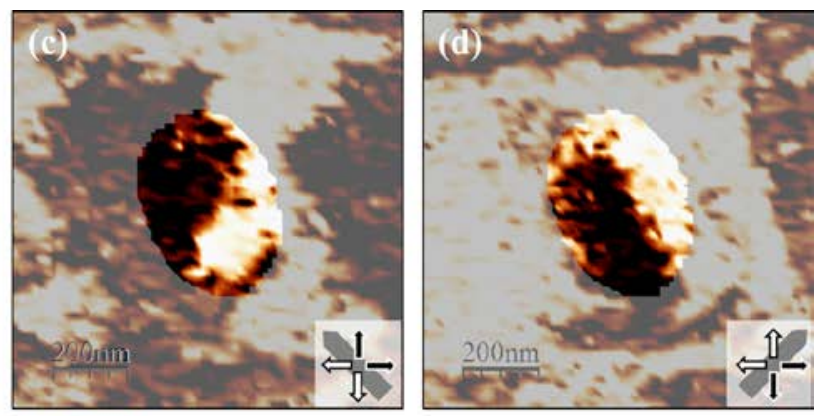

(f)

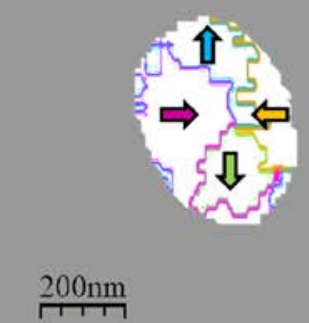

Figure 4. Topography (a), Vertical PFM phase (b), Lateral PFM phase with probe along [0-10] (c) and [-100] (d), Vector PFM (e) with planar colour coding (left) and a schematic of the domain structure (f) of an island after switching by positive voltage from the bottom electrode. A topographic mask is superimposed onto the PFM images and Vector PFM maps in order to identify the island position. Inset diagrams in (c) and (d) represent the cantilever orientation with color coding for Lateral PFM image interpretation.

Following polarization reversal by positive bias (which for brevity we will refer to as the 'reset' operation), scanning with negative DC bias (typically -6V) induced domain configurations which were almost entirely dependent on the slow raster axis direction used. Poling with the probe scanning along [100] or [010] led to multidomain configurations (Figure 
S3) similar to the stripe-like configurations resulting from switching experiments on BFO film surfaces where all polarization variants are allowed ${ }^{21}$. However, poling with the slow raster axis along the surface projection of one of the polar axes (Figure 2a), yielded domain configurations with almost a single domain variant (Figure 5a). All four polarization variants with downwards out-of-plane components could be selected. For instance, poling with slow raster scan axis along [110] creates an island with a predominant [-1-1-1] polar orientation (Figure 5a, bottom-right image). Analysis of domain populations (Figure 5b) show deterministic selection of the desired variant on at least $70 \%$ of the area of the island. Notably, areas not displaying the selected variant are mostly located at island sidewalls; sidewalls have been subjected to an ion dose during the fabrication procedure, and it is known that this can affect the ferroelectric properties of the material. When only the very top surface of the island is considered, then at least $90 \%$ of the domains observed are seen to be deterministically selected, while less control occurs at the sidewalls (Figure S8).

The procedure is repeatable, reproducible and leads to domain configurations with good retention (Figure S9). Islands can be switched among any of the four down-state variants, after a 'reset' operation, by applying negative DC bias combined with a suitable slow scan direction. It has to be noted that application of negative bias on an island with out-of-plane component already downwards (i.e. without previous 'reset' biasing) doesn't induce any domain reconfiguration. Hence, it can be inferred that the influence of the trailing field can only be realized in conjunction with reversal of the out-of-plane polarization. 


\section{(a)}

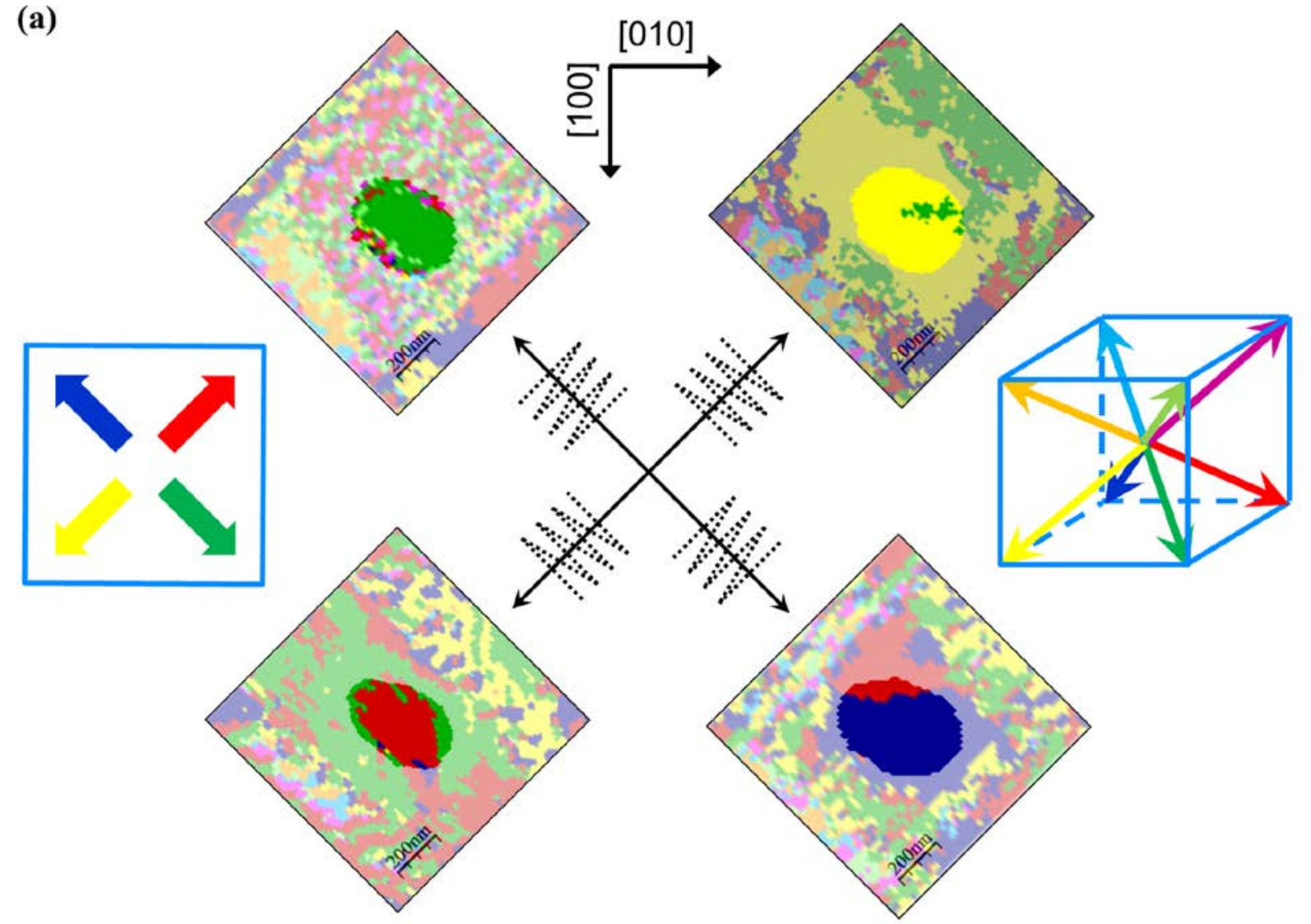

(b)

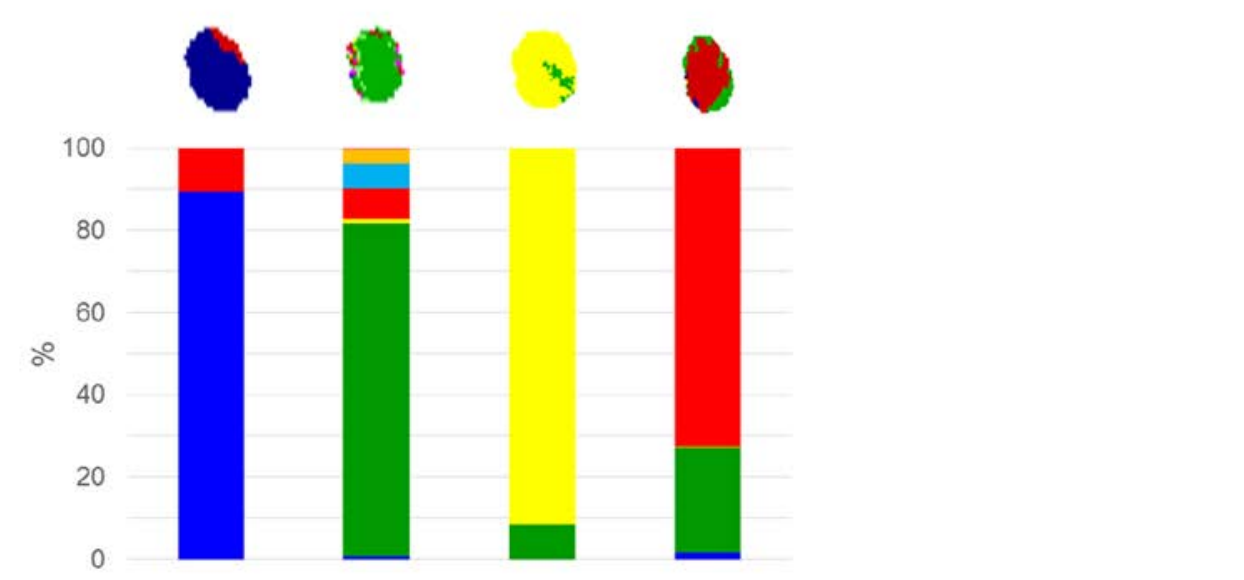

Figure 5. Vector PFM images resulting after switching experiments (typically $-6 \mathrm{~V}$ ) with the slow raster axis for each image indicated in the centre of the diagram (a) and stacked column charts of the domain population after each switching experiment (b). The obtained variant is unequivocally determined by the slow raster axis direction. Planar color coding ((a) top left) for 
polar orientations as viewed from the BFO surface, and a diagram of the BFO unit cell with its $<111>_{\text {pseudocubic }}$ polarization variants ((a) top right).

In order to gain more insight as to the nature of the 'trailing field' responsible for the deterministic selection of polarization variants, we considered the electric field in the system using finite element modelling (Quickfield). The film thickness was set to $40 \mathrm{~nm}$, with bias applied from the bottom electrode and, considering the rhombohedral structure of BFO, we assumed an isotropic dielectric permittivity with a set value of $\varepsilon=100^{35}$. We modeled the PFM conductive probe in contact with the surface as a 10nm top capacitor. This was consistent with a tip-surface contact diameter in the 'strong indentation regime' (the ideal condition for performing $\mathrm{PFM}^{36}$ ). The outcome of the simulation is displayed in Figure 6, showing a field distribution with a relatively homogeneous normal component (Figure 6a) as well as distinct localized transverse components (Figure 6b). Fringing fields at the edges of the tip-sample contact are clear. Figure 6c shows that the transverse fields at the surface can be relatively intense, but decrease rapidly away from the surface (Figure 6c, open symbols). High transverse field components therefore seem to be confined into small volumes at the edges of the contact area.

Even assuming transverse fields high enough to reorient the in-plane polarization component when used as a trailing field, this would take place only in a confined volume very close to the surface. Any possible transverse field-driven reorientation taking place would not propagate through the sample's thickness and, as a consequence, would not be stable, leading to limited domain reorganization. On the other hand, poling (after reset biasing) leads to out-of-plane reorientation. The four possible variants are nearly energetically equivalent and hence even a 
small in-plane field (the direction of which is determined by the probe's movement during the operation) effectively selects one of the four available polarization variants.

(a)

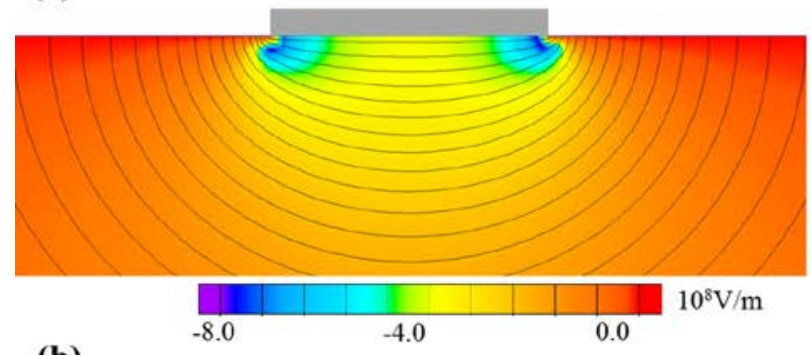

(b)

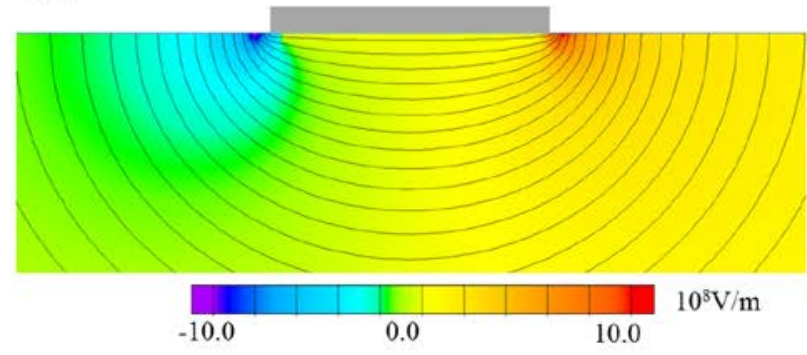

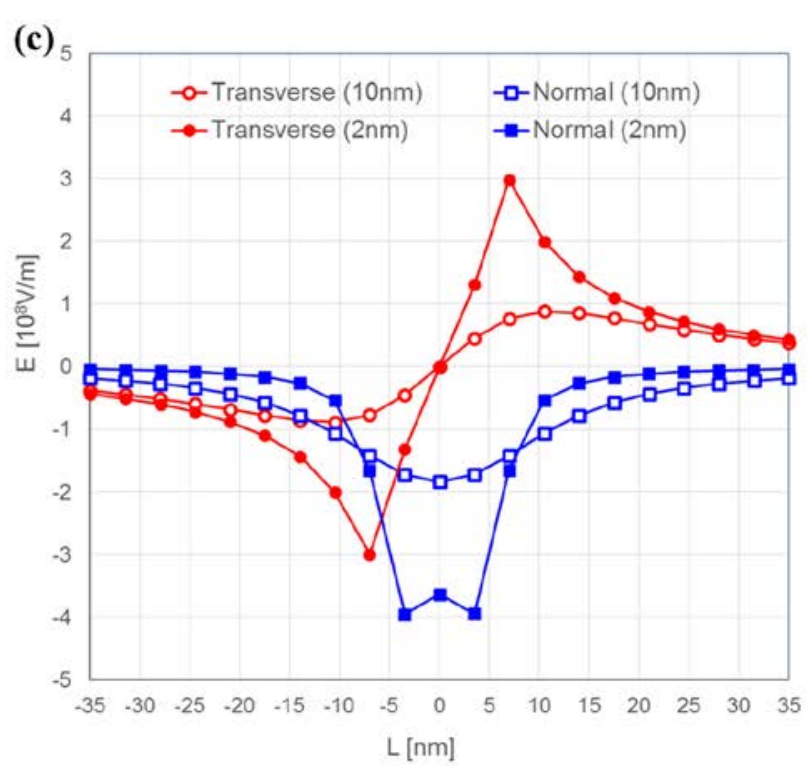

Figure 6. Normal (a) and transverse (b) electrical field component as determined by finite element modelling. A $-6 \mathrm{~V}$ bias potential was set at the bottom electrode and dropped across a BFO thin film ( $\varepsilon=100,40 \mathrm{~nm}$ thickness), with a conductive scanning probe tip grounded. The tip was modeled as a top electrode (10nm diameter). Line profiles for normal (square symbols) and transverse field (round symbols), 2nm and 10nm (closed and open symbols respectively) below the BFO film surface (c).

It should be finally noted that the lack of any domain reconfiguration without prior reset poling might seem to contradict previously reported results ${ }^{22}$, in which domain alterations could be obtained even in absence of out-of-plane polar reversal. However, in this previous research the high voltages involved were thought to induce localised phase transitions. In the present case, the applied bias is just enough to switch polarization; no field-induced phase transitions are expected. 
In conclusion, switching to obtain specific polarization variants in (001) BFO nanoislands has been experimentally demonstrated. We have shown that each of the four available variants with out-of-plane components pointing downwards can be independently selected depending on the sense of the 'trailing' field associated with a moving PFM tip. Electric field modeling reveals the presence of high transverse fields within small volumes at the edges of the probe-surface contact area and it appears to be these fields that are responsible for polar variant selection, during the out-of-plane polarization reversal process.

This work demonstrates that a higher degree of control on functional properties can be achieved in nanostructures, due to reduced dimensionality and the lack of constraint from surrounding matrix material. Selection of polarization variants will be of use for verification of electric field driven magnetization switching in BFO-based multiferroic heterostructures with nanoscale lateral size, and therefore the research may contribute towards the realization of magnetoelectric memories.

\section{ASSOCIATED CONTENT}

Supporting Information. Piezo-hysteresis loops; full Piezoresponse Force Microscopy data for Figures 4 and 5; domain population analysis; Piezoresponse Force Microscopy imaging for additional switching experiments; retention data. This material is available free of charge via the Internet at http://pubs.acs.org.

\section{AUTHOR INFORMATION}

\section{Corresponding Author}

* Email: alessio.morelli.pfm@gmail.com 


\section{Present Addresses}

$\S$ Asylum Research, an Oxford Instruments company, Borsigstrasse 15a, 65205 Wiesbaden, Germany

\| School of Materials Science and Engineering, University of New South Wales, Sydney NSW 2052, Australia

\section{Author Contributions}

The manuscript was written through contributions of all authors. All authors have given approval to the final version of the manuscript.

\section{Funding Sources}

FP7-PEOPLE-2013-IEF, Grant Agreement PIEF-GA-2013-623524

Notes

Any additional relevant notes should be placed here.

\section{ACKNOWLEDGMENT}

The samples used for the present research were grown at the Max-Planck Institute of Microstructure Physics in Halle an der Saale (DE), for which we acknowledge the assistance and supervision of Ionela Vrejoiu. The research leading to these results has received funding from the People Programme (Marie Curie Actions) of the European Union's Seventh Framework Programme (FP7/2007-2013) under REA grant agreement nº [PIEF-GA-2013-623524].

\section{REFERENCES}

(1) Waldrop, M. M. Nature 2016, 530, 144-147. 
(2) Kleemann, W. Physics 2009, 2, 105.

(3) Eerenstein, W.; Mathur, N. D.; Scott, J. F. Nature 2006, 442, 759-765.

(4) Fiebig, M. J. Phys. D: Appl. Phys. 2005, 38 (8), R123-R152.

(5) Ma, J.; Hu, J.; Li, Z.; Nan, C.-W. Adv. Mater. 2011, 23 (9), 1062-1087.

(6) Pyatakov, A. P.; Zvezdin, A. K. Phys.-Usp. 2012, 55 (6), 557-581.

(7) Vaz, C. A. F. J. Phys.: Condens. Matter 2012, 24 (33), 333201.

(8) Catalan, G.; Scott, J. F. Adv. Mater. 2009, 21 (24), 2463-2485.

(9) Chu, Y. H.; Martin, L. W.; Holcomb, M. B.; Gajek, M.; Han, S. J.; He, Q.; Balke, N.; Yang, C. H.; Lee, D.; Hu, W.; Zhan, Q.; Yang, P. L.; Fraile-Rodriguez, A.; Scholl, A.; Wang, S. X.; Ramesh, R. Nat. Mater. 2008, 7 (8), 678.

(10) Lebeugle, D.; Mougin, A.; Viret, M.; Colson, D.; Ranno, L. Phys. Rev. Lett. 2009, 103 (25), 257601.

(11) Heron, J.; Trassin, M.; Ashraf, K.; Gajek, M.; He, Q.; Yang, S.; Nikonov, D.; Chu, Y.-H.; Salahuddin, S.; Ramesh, R. Phys. Rev. Lett. 2011, 107 (21), 217202.

(12) Heron, J. T.; Bosse, J. L.; He, Q.; Gao, Y.; Trassin, M.; Ye, L.; Clarkson, J. D.; Wang, C.; Liu, J.; Salahuddin, S.; Ralph, D. C.; Schlom, D. G.; Iniguez, J.; Huey, B. D.; Ramesh, R. Nature 2014, 516, 370-373.

(13) Zhao, T.; Scholl, A.; Zavaliche, F.; Lee, K.; Barry, M.; Doran, A.; Cruz, M. P.; Chu, Y. H.; Ederer, C.; Spaldin, N. A.; Das, R. R.; Kim, D. M.; Baek, S. H.; Eom, C. B.; Ramesh, 
R. Nat. Mater. 2006, 5 (10), 823-829.

(14) Martin, L. W.; Chu, Y.-H.; Holcomb, M. B.; Huijben, M.; Yu, P.; Han, S.-J.; Lee, D.; Wang, S. X.; Ramesh, R. Nano Lett. 2008, 8 (7), 2050-2055.

(15) Heron, J. T.; Schlom, D. G.; Ramesh, R. Appl. Phys. Rev. 2014, 1 (2), 021303.

(16) Kubel, F.; Schmid, H. Acta Crystallogr., Sect. B 1990, 46 (6), 698-702.

(17) Baek, S. H.; Jang, H. W.; Folkman, C. M.; Li, Y. L.; Winchester, B.; Zhang, J. X.; He, Q.; Chu, Y. H.; Nelson, C. T.; Rzchowski, M. S.; Pan, X. Q.; Ramesh, R.; Chen, L. Q.; Eom, C. B. Nat. Mater. 2010, 9 (4), 309-314 .

(18) Vasudevan, R. K.; Liu, Y. Y.; Li, J. Y.; Liang, W. I.; Kumar, A.; Jesse, S.; Chen, Y. C.; Chu, Y. H.; Nagarajan, V.; Kalinin, S. V. Nano Lett. 2011, 11 (8), 3346-3354.

(19) Matzen, S.; Nesterov, O.; Rispens, G.; Heuver, J. A.; Biegalski, M.; Christen, H. M.; Noheda, B. Nat. Commun. 2014, 5, 4415.

(20) Wang, C.; Ke, X.; Wang, J.; Liang, R.; Luo, Z.; Tian, Y.; Yi, D.; Zhang, Q.; Wang, J.; Han, X.-F.; Van Tendeloo, G.; Chen, L.-Q.; Nan, C.-W.; Ramesh, R.; Zhang, J. Nat. Commun. 2016, 7, 10636.

(21) Balke, N.; Choudhury, S.; Jesse, S.; Huijben, M.; Chu, Y. H.; Baddorf, A. P.; Chen, L. Q.; Ramesh, R.; Kalinin, S. V. Nat. Nanotechnol. 2009, 4 (12), 868-875.

(22) Bea, H.; Ziegler, B.; Bibes, M.; Barthelemy, A.; Paruch, P. J. Phys.: Condens. Matter 2011, 23 (14), 142201. 
(23) Crassous, A.; Sluka, T.; Tagantsev, A. K.; Setter, N. Nat. Nanotechnol. 2015, 10 (7), 614 618.

(24) Johann, F.; Morelli, A.; Biggemann, D.; Arredondo, M.; Vrejoiu, I. Phys. Rev. B 2011, 84 (9), 94105.

(25) Morelli, A.; Johann, F.; Schammelt, N.; McGrouther, D.; Vrejoiu, I. J. Appl. Phys. 2013, 113 (15), 154101.

(26) Morelli, A.; Vrejoiu, I. Epitaxial Ferroelectric Nanostructures Fabricated by FIB Milling. In FIB Nanostructures; Wang, Z. M., Ed.; Lecture Notes in Nanoscale Science and Technology; Springer International Publishing: Cham, 2013; Vol. 20, pp 23-43.

(27) Rodriguez, B. J.; Gruverman, A.; Kingon, A. I.; Nemanich, R. J.; Cross, J. S. Appl. Phys. A 2004, 80 (1), 99-103.

(28) Harnagea, C.; Pignolet, A.; Alexe, M.; Hesse, D.; Gösele, U. Appl. Phys. A 2000, 70, 261267.

(29) Ivry, Y.; Chu, D. P.; Scott, J. F.; Durkan, C. Phys. Rev. Lett. 2010, 104 (20), 207602.

(30) McGilly, L. J.; Schilling, A.; Gregg, J. M. Nano Lett. 2010, 10 (10), 4200-4205.

(31) McGilly, L. J.; Gregg, J. M. Appl. Phys. Lett. 2011, 98 (13), 132902.

(32) Vasudevan, R. K.; Chen, Y.-C.; Tai, H.-H.; Balke, N.; Wu, P.; Bhattacharya, S.; Chen, L. Q.; Chu, Y.-H.; Lin, I.-N.; Kalinin, S. V; Nagarajan, V. ACS Nano 2011, 5 (2), 879-887.

(33) Chu, Y.-H.; He, Q.; Yang, C.-H.; Yu, P.; Martin, L. W.; Shafer, P.; Ramesh, R. Nano Lett. 
2009, 9 (4), 1726-1730.

(34) Grossmann, M.; Lohse, O.; Bolten, D.; Boettger, U.; Waser, R. J. Appl. Phys. 2002, 92 (5), 2688.

(35) Lobo, R. P. S. M.; Moreira, R. L.; Lebeugle, D.; Colson, D. Phys. Rev. B 2007, 76 (17), 172105.

(36) Kalinin, S. V.; Bonnell, D. Phys. Rev. B 2002, 65 (12), 125408.

Insert Table of Contents Graphic and Synopsis Here

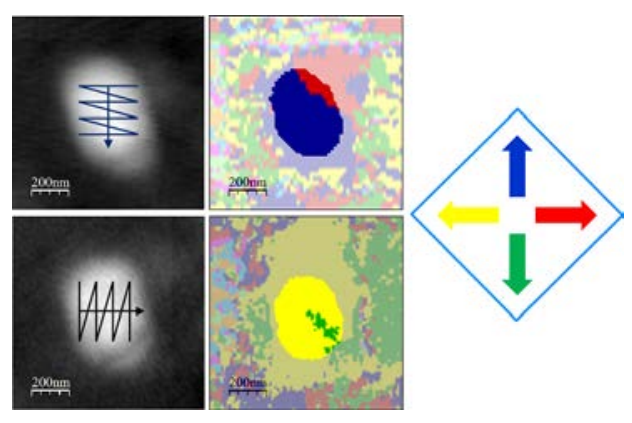

Deterministic polarization switching: the desired variant is selected by raster scanning direction during switching procedure. 